\title{
Fischer-Tropsch Refining
}

by

\section{Arno de Klerk}

A thesis submitted in partial fulfillment

of the requirements for the degree

\section{Philosophiae Doctor (Chemical Engineering)}

in the

\author{
Department of Chemical Engineering
}

Faculty of Engineering, the Built Environment and Information Technology

\author{
University of Pretoria \\ Pretoria
}

South Africa

February 2008 


\section{Fischer-Tropsch Refining}

Author: Arno de Klerk

Promoter: Prof. Philip L. de Vaal

Department: Department of Chemical Engineering, University of Pretoria

Degree: Philosophiae Doctor (Chemical Engineering)

\section{Synopsis}

Energy carriers, such as coal, natural gas and biomass, can be converted by Fischer-Tropsch technology into synthetic crude (syncrude). Fischer-Tropsch derived syncrude can then be refined to transportation fuels, such as motor-gasoline, jet fuel and diesel fuel. These fuels meet the same specifications as crude oil derived transportation fuels.

Conventional refining technologies have to be adapted to deal with Fischer-Tropsch syncrudes, because they differ significantly from crude oil with respect to composition. Some of the key differences are the high concentration of oxygenates and olefins and absence of sulphur in Fischer-Tropsch syncrude. Imposing a crude oil refining methodology on syncrude can lead to unwieldy and expensive refineries. Yet, despite an abundance of literature of Fischer-Tropsch synthesis, there is little literature that deals with the refining of Fischer-Tropsch syncrude.

The present study investigated current refining practice for both crude oil and FischerTropsch syncrude in order to identify fundamental differences in their refining focus and conversion behaviour. This was followed by a critical evaluation of the compatibility of syncrudes from high temperature Fischer-Tropsch (HTFT) and low temperature FischerTropsch (LTFT) synthesis with the chemistry and catalysis of various conversion processes. The conversion processes that were evaluated include isomerisation, oligomerisation, etherification, alkylation, metathesis, hydrogenation, hydroisomerisation, hydrocracking, catalytic cracking, coking, thermal cracking, catalytic reforming and dehydration. The recommendations from the technology evaluation provided the foundation for the development of Fischer-Tropsch syncrude based refinery designs.

Refinery designs were developed to determine configurations that would maximise the production of on-specification motor-gasoline, jet fuel and diesel fuel respectively. It 
could be shown that less complex refinery designs were required to refine Fischer-Tropsch syncrude to motor-gasoline and jet fuel, than were required for crude oil refining. It was also shown that on a molecular level Fischer-Tropsch syncrude is unsuited for maximising the production of Euro-4 type diesel fuel.

The present study illustrates the advantage of considering fundamentals in developing refineries specifically for Fischer-Tropsch syncrude, rather than imposing crude oil design practises on Fischer-Tropsch syncrude refinery designs.

Keywords: Fischer-Tropsch, refining, syncrude, motor-gasoline, jet fuel, diesel fuel, refinery design, refining catalysis. 


\section{Acknowledgements}

I am indebted to my wife, Chèrie, for her love, support and encouragement. 


\section{Table of Contents}

\section{Chapter I - Prologue}

1. Introduction 1

1.1. $\quad$ Crude oil for transportation fuel 1

1.2. Energy insecurity 6

1.3. Fischer-Tropsch technology 7

1.4. Refining of Fischer-Tropsch products 10

2. Justification 11

2.1. Academic justification 11

2.2. Industrial justification 12

3. Aim and scope 12

3.1. Thesis part 1: Background 13

3.2. Thesis part 2: Refining technology selection and refinery design 13

4. Literature cited 13

\section{Chapter II - Fuel Specifications}

1. Introduction 17

2. Motor-gasoline (petrol) 19

2.1. International motor-gasoline specifications 20

2.2. Motor-gasoline properties 23

2.2.1. Octane number 23

2.2.2. Volatility 25

2.2.3. Density 26

2.2.4. Oxygenate content 26

2.2.5. Olefin content 27

2.2.6. Aromatics content 27

2.2.7. Sulphur content 28

2.2.8. Metal content $\quad 29$

2.2.9. Oxidation stability and gum formation 30

3. Diesel 31

3.1. International diesel specifications 32 
3.2. Diesel properties 34

3.2.1. Cetane number 34

3.2.2. Flash point 36

3.2.3. Density and viscosity 36

3.2.4. Aromatics content 37

3.2.5. Sulphur content 37

3.2.6. Lubricity 38

3.2.7. Cold flow properties $\quad 40$

4. Aviation turbine fuel (jet fuel) 40

4.1. International specifications $\quad 41$

4.2. Aviation turbine fuel properties 43

4.2.1. Heat of combustion 43

4.2.2. Density and viscosity 44

4.2.3. Aromatic content and smoke point 45

4.2.4. Sulphur content $\quad 45$

4.2.5. Freezing point $\quad 46$

4.2.6. Volatility 46

4.2.7. Thermal stability $\quad 46$

5. Trends for future fuel specifications $\quad 47$

5.1. $\quad$ Future motor-gasoline 47

$\begin{array}{lll}\text { 5.2. } & \text { Future diesel } & 48\end{array}$

5.3. Future aviation turbine fuel 49

6. Literature cited 50

A. $\quad$ Origin of the European Euro-3 and Euro-4 fuel specifications 57

B. Theoretical limitations and implications of petrol specifications 59

\section{Chapter III - Crude Oil}

1. Introduction 62

$\begin{array}{lll}2 . & \text { Crude oil composition } & 63\end{array}$

2.1. Hydrocarbons 64

2.2. Sulphur containing compounds 65

2.3. Oxygen containing compounds 66

2.4. Nitrogen containing compounds 68 
2.5. Metal containing compounds

69

3. Crude oil physical properties $\quad 70$

3.1. Density 70

$\begin{array}{lll}\text { 3.2. Pour point } & 70\end{array}$

3.3. Viscosity 71

3.4. Vapour pressure $\quad 71$

$\begin{array}{lll}\text { 3.5. Distillation } & 71\end{array}$

$\begin{array}{lll}\text { 4. } & \text { Literature cited } & 73\end{array}$

\section{Chapter IV - Crude Oil Refineries}

$\begin{array}{lll}\text { 1. Introduction } & 75\end{array}$

2. Separation processes 81

3. Conversion processes $\quad 84$

3.1. Residue upgrading 85

3.1.1. Conversion of residue to fuels 85

3.1.2. Non-fuels application of residues 86

3.2. Diesel and jet fuel upgrading 86

3.3. Naphtha and gas upgrading $\quad 87$

4. Future crude oil refineries 90

4.1. Change drivers in crude oil refining $\quad 90$

4.2. Changes in crude oil refining 91

4.3. Future crude oil refineries 93

$\begin{array}{lll}\text { 5. } & \text { Literature cited } & 95\end{array}$

\section{Chapter V - Fischer-Tropsch Syncrude}

1. Introduction 99

2. Fischer-Tropsch catalysis 100

$\begin{array}{lll}\text { 2.1. Catalyst properties } & 102\end{array}$

2.1.1. Probability of chain growth 102

2.1.2. Hydrogenation activity 103

$\begin{array}{ll}\text { 2.1.3. Water gas shift activity } & 104\end{array}$

2.1.4. Sensitivity to promoters 104

$\begin{array}{lll}\text { 2.2. Influence of operating conditions } & 105\end{array}$ 
2.2.1. Synthesis gas composition

2.2.2. Pressure

2.2.3. Temperature

3. Syncrude composition 106

3.1. Hydrocarbons 107

$\begin{array}{lll}\text { 3.2. Oxygenates } & 108\end{array}$

3.3. Metal containing compounds 110

4. Properties of commercial syncrudes 111

5. Comparison of crude oil and syncrude 112

6. Literature cited 115

\section{Chapter VI - Fischer-Tropsch Refineries}

$\begin{array}{lll}1 . & \text { Introduction } & 118\end{array}$

2. German technology (1930-1940’s) 119

2.1. Normal-pressure cobalt Fischer-Tropsch synthesis 120

2.2. Refining of normal-pressure syncrude 123

3. United States technology (1940-1950’s) 126

3.1. Hydrocol Fischer-Tropsch synthesis 127

3.2. Refining of Hydrocol syncrude 129

4. Sasol 1 technology (1950’s) 131

4.1. Kellogg Fischer-Tropsch synthesis 132

4.2. Arge Fischer-Tropsch synthesis 136

$\begin{array}{lll}\text { 4.3. } & \text { Sasol } 1 \text { gas loop } & 137\end{array}$

4.4. Sasol 1 refinery $\quad 141$

4.4.1. Sasol 1 tar work-up $\quad 142$

4.4.2. Sasol 1 Kellogg oil work-up 143

4.4.3. Sasol 1 Arge oil work-up $\quad 144$

4.4.4. Sasol 1 chemical work-up $\quad 146$

5. South African Sasol 2 and 3 technology (1970-1980’s) 147

5.1. Sasol Synthol Fischer-Tropsch synthesis 148

5.2. Sasol 2 and 3 gas loops 150

5.3. Sasol 2 and 3 refineries 153

5.3.1. Sasol 2 and 3 tar work-up $\quad 155$ 
5.3.2. Sasol 2 and 3 condensate and oil work-up $\quad 156$

5.3.3. Sasol 2 and 3 chemical work-up $\quad 160$

6. Mossgas gas-to-liquids technology (1980-1990’s) 161

6.1. Mossgas Fischer-Tropsch synthesis 162

6.2. Mossgas gas loop 162

6.3. Mossgas refinery 165

6.3.1. Mossgas oil and condensate work-up 165

$\begin{array}{lll}\text { 6.3.2. } & \text { Mossgas chemical work-up } & 167\end{array}$

7. Shell gas-to-liquids technology (1980-1990’s) 167

7.1. Shell Bintulu Fischer-Tropsch synthesis 168

7.2. Shell Bintulu gas loop 169

7.3. Shell Bintulu refinery 169

8. Sasol gas-to-liquids technology (2000’s) 170

8.1. $\quad$ Oryx GTL Fischer-Tropsch synthesis 171

8.2. Oryx GTL gas loop 171

8.3. Oryx GTL refinery 173

9. Evolution of Sasol Fischer-Tropsch refineries 174

9.1. Evolution of Sasol $1 \quad 175$

$\begin{array}{lll}\text { 9.2. } & \text { Evolution of Sasol } 2 \text { and } 3 & 178\end{array}$

10. Future Fischer-Tropsch refineries 184

10.1. Change drivers in Fischer-Tropsch refining 184

10.2. Design of future Fischer-Tropsch refineries 186

11. Literature cited 186

Chapter VII - Refining technologies evaluated in Fischer-Tropsch context

1. Introduction 196

$\begin{array}{lll}2 . & \text { Olefin conversion } & 197\end{array}$

2.1. Double bond isomerisation 197

2.2. Oligomerisation 199

2.3. Olefin skeletal isomerisation 206

2.4. Etherification 208

$\begin{array}{ll}\text { 2.5. Aliphatic alkylation } & 210\end{array}$ 
2.6. Aromatic alkylation 212

$\begin{array}{lll}\text { 2.7. } & \text { Metathesis } & 214\end{array}$

3. Hydrogen addition 215

3.1. Hydrotreating 215

$\begin{array}{ll}\text { 3.2. Hydroisomerisation } & 217\end{array}$

3.3. Hydrocracking 220

4. Carbon rejection 222

4.1. Fluid catalytic cracking 222

4.2. Coking 225

5. Hydrogen rejection 227

5.1. Thermal cracking 227

5.2. Catalytic reforming 229

5.3. Aromatisation 231

5.4. Alcohol dehydration 232

6. Discussion 234

$\begin{array}{ll}\text { 7. } & \text { Literature cited } \\ & 237\end{array}$

\section{Chapter VIII - Refinery design}

1. Introduction 254

2. Conceptual refinery design 256

3. Real-world refinery design 258

$\begin{array}{ll}\text { 3.1. } & \text { Refinery type } 258\end{array}$

3.2. Products and markets 259

$\begin{array}{lll}\text { 3.3. Feedstock } & 259\end{array}$

3.4. Location 261

3.5. Secondary design objectives 264

3.6 Other issues 266

$\begin{array}{lll}\text { 4. } & \text { Literature cited } & 267\end{array}$

Chapter IX - Conceptual Fischer-Tropsch refinery designs

1. Introduction 269

2. Modelling details 270

2.1. Conceptual design 270 
2.2. Refinery economics 271

3. Motor-gasoline refineries 272

3.1. HTFT motor-gasoline refinery development 275

3.1.1. HTFT paraffinic motor-gasoline 278

3.1.2. HTFT aromatic motor-gasoline 281

3.1.3. HTFT olefinic motor-gasoline 283

3.1.4. HTFT oxygenated motor-gasoline 283

3.2. HTFT motor-gasoline refinery flowschemes 284

$\begin{array}{lll}\text { 3.2.1. } & \text { Flowscheme } 1 & 284\end{array}$

3.2.2. Flowscheme $2 \quad 287$

3.2.3. Flowscheme $3 \quad 291$

3.3. LTFT motor-gasoline refinery development 294

3.3.1. Catalytic cracking of LTFT wax 295

3.3.2. Hydrocracking of LTFT wax 296

3.4. LTFT motor-gasoline refinery flowschemes 297

3.4.1. Flowscheme $4 \quad 297$

3.4.2. Flowscheme $5 \quad 300$

3.4.3. Flowscheme $6 \quad 302$

4. Jet fuel refineries 305

4.1. HTFT jet fuel refinery development 307

4.2. HTFT jet fuel refinery flowschemes 309

$\begin{array}{lll}\text { 4.2.1. } & \text { Flowscheme } 7 & 309\end{array}$

4.2.2. Flowscheme $8 \quad 312$

4.2.3. Flowscheme $9 \quad 314$

4.3. LTFT jet fuel refinery development 316

4.4. LTFT jet fuel refinery flowschemes 317

4.4.1. Flowscheme $10 \quad 317$

5. Diesel fuel refineries 320

5.1. HTFT diesel fuel refinery development 325

5.2. HTFT diesel fuel refinery flowschemes 327

5.2.1. Flowscheme $11 \quad 327$

5.2.2. Flowscheme $12 \quad 330$

5.2.3. Flowscheme 13 
5.3. LTFT diesel fuel refinery development 335

5.4. $\quad$ LTFT diesel fuel refinery flowschemes 338

5.4.1. Flowscheme $14 \quad 338$

5.4.2. Flowscheme $15 \quad 340$

5.4.3. Flowscheme $16 \quad 342$

6. Literature cited 345

A. Design basis for conceptual refinery development 349

\section{Chapter X - Conclusion}

1. Introduction 356

2. What has been achieved? 357

2.1. Thesis part 1: Background 357

2.2. Thesis part 2: Refining technology and refinery design 358

3. Prospects for future study 359 